\title{
Improving light curve parameters of exoplanets based on TESS data
}

\author{
P. Gajdoš ${ }^{1}$, A. Maliuk ${ }^{2}$, M. Vítková ${ }^{3}$ and H. Parviainen ${ }^{4,5}$ \\ 1 Institute of Physics, Faculty of Science, Pavol Jozef Šafárik University, \\ 04001 Košice, Slovakia (E-mail: pavol.gajdos@student.upjs.sk) \\ 2 Astronomical Institute of the Slovak Academy of Sciences \\ 05960 Tatranská Lomnica, The Slovak Republic(E-mail: amaliuk@ta3.sk) \\ 3 Department of Theoretical Physics and Astrophycis, Masaryk University, \\ Kotlárská 2, 61137 Brno, Czech Republic (E-mail: 474624@mail.muni.cz) \\ ${ }^{4}$ Instituto de Astrofísica de Canarias (IAC), 38205 La Laguna, Tenerife, \\ Spain \\ 5 Departamento de Astrofísica, Universidad de La Laguna (ULL), 38206, La \\ Laguna, Tenerife, Spain
}

Received: September 15, 2020; Accepted: October 16, 2020

\begin{abstract}
We revise parameters of four transiting hot Jupiter planets discovered by ground-based surveys. We use follow-up observations carried by TESS mission together with PyTransit software. Space telescopes like TESS provide better coverage and photometry precision than ground-based telescopes. PyTransit is fast and user-friendly exoplanet transit light curve modelling package, implementing optimised versions of the Gimenéz and the Mandel \& Agol transit models. After applying PyTransit to TESS data, our results show an improvement in the exoplanets parameters estimation.
\end{abstract}

Key words: Methods: data analysis - Techniques: photometric - Stars: planetary systems - Stars: individual: HAT-P-5, HATS-33, HATS-24

\section{Introduction}

Extrasolar planets are exotic substellar objects which were discovered only recently. This field of research is evolving quickly and opening new possibilities, surprises and challenges.

There are different methods for detecting exoplanets. The major part of exoplanets was discovered using transit and radial velocity methods. Transit photometry is based on decreasing of stellar brightness while exoplanet moves across the disc of the host star. Planets that eclipse their host star during their orbit are key objects for the study of exoplanetary systems. Our main goal is to improve the precision of known exoplanet parameters using new data from the Transiting Exoplanet Survey Satellite (TESS).

The TESS, launched in 2018, was designed as all-sky space survey searching for exoplanets orbiting the bright stars - brighter than 12 mag (Ricker et al., 
2014). The spacecraft consists of four 100-mm telescopes (f/1.4) with four CCD cameras each with resolution $4 \mathrm{Mpx}$. The complete field of view is $24 \times 96$ degrees. TESS uses the broad bandpass filter $(600-1000 \mathrm{~nm})$ which is centred on the traditional $I_{\mathrm{C}}$ filter. During the primary mission (from July 2018 to July 2020), the nearly whole sky was observed in 26 sectors. Each sector is 27.4-days long. Every 30 minutes, the full-frame image was obtained. For selected targets, the short-cadence data is available. They are collected every 2 minutes.

Up to date (24 September 2020) ${ }^{1}$, 74 confirmed planets and more than 2300 candidates were discovered by TESS. The first exoplanet discovered by TESS is Super-Earth $\pi$ Mensae c (Huang et al., 2018). At the beginning of 2020, the discovery of the first Earth-sized exoplanet in the habitable zone TOI-700 d was reported (Gilbert et al., 2020). Vanderburg et al. (2020) recently, in September 2020, presented the planet candidate (TIC 267574918) transiting a white dwarf. Many of exoplanets discovered by other missions were already observed by TESS with very high precision which allows us to analyse these planets in more details. Compared with most of the ground-based surveys, TESS provides much better coverage and photometry precision. This allows us to collect more reliable data about known exoplanets.

In this report, we summarised our results obtained during "GAIA \& TESS: Tools for understanding of the Local Universe" summer school. We used the data obtained by the TESS mission to study transiting exoplanets. Our main task was to study the already known transiting exoplanets using the TESS data and compare the obtained values of parameters with the literature.

\section{Transit Modelling}

During our work on exoplanet characterisation, we used PyTransit software created by Parviainen (2015). PyTransit is a package for exoplanet transit light curve modelling. PyTransit combines a Bayesian approach to inference with Markov chain Monte Carlo (MCMC) sampling for the posterior estimation. This allows improving characterisation of model parameters uncertainties. The package includes necessary utility routines to calculate circular or elliptic orbits, transit durations, eclipse centres etc. PyTransit needs normalised de-trended light curve for work. Some small additional trend and/or stellar variability could be removed using gaussian processes (Foreman-Mackey et al., 2017) during the fitting. Initial distributions of prior parameters (orbital period, time of primary transit, transit depth) are also required. The package can use Giménez (2006), Mandel \& Agol (2002), Maxted \& Gill (2019) or RoadRunner (Parviainen, 2020) transits models with various limb darkening laws.

The Bayesian approach allows finding posterior probability density of target parameters if likelihood function and prior density are known. This method can be implied iteratively after each new piece of data arrives. This allows us to

\footnotetext{
${ }^{1}$ https://exoplanetarchive.ipac.caltech.edu/
} 
use known exoplanet parameters from the literature as priors for PyTransit. From previous studies, we use transit depth, orbital period and time of primary transit as initial values which were optimized with other parameters during the fitting.

In this work, we use Mandel \& Agol transit model with quadratic limb darkening law as the most common one. Limb darkening coefficients were optimised by PyTransit. We assume that the shapes of the planet and star are spherical and orbits are circular. The algorithm operates by phase-folding data over a range of trial parameters (transit depth, orbital period, time of primary transit, impact parameter, stellar density and limb darkening coefficients). It calculates the $\chi^{2}$ statistic of the phase-folded light curve between the data points of the respective transit model and the observed values. Then the global $\chi^{2}$ minimum is used. Markov chain Monte Carlo methods create samples from a continuous random variable, with probability density proportional to a known function. Creating synthetic light curves with this method and running the model fitting on them gives us a reliable estimation of uncertainties of parameters of our model. Finally, we calculated the values of some common planetary parameters (semi-major axis, radius ratio, planet radius and inclination) from the obtained values of optimized parameters (mentioned above).

\section{Studied systems}

For the purpose of this study, we selected three transiting exoplanets (see Tab. 1) discovered by ground-based projects HAT (Hungarian Automated Telescope Network; Bakos et al., 2004) and HAT South (Bakos et al., 2013). These targets were already observed by TESS mission and have not been studied using these data, yet. The selected planets belong to the category of hot Jupiter planets. These are Jupiter-size planets orbit the parent star on very close orbits with periods of only a few days.

\subsection{HAT-P-5 b}

The hot Jupiter HAT-P-5 b was discovered by HATNet project in 2005 and confirmed in 2007 by Bakos et al. (2007). This exoplanet was named by Slovak citizens in IAU campaign "NameExoWorlds" (Penteado et al., 2019). The name of the planet is Králomoc and parent star is Chason. The names come from Slavic mythology. The parent star is a sun-like star with effective temperature $5960 \pm 100 \mathrm{~K}$, radius $1.12 \pm 0.09 \mathrm{R}_{\odot}$ and mass $1.04 \pm 0.29 \mathrm{M}_{\odot}$ (Stassun et al., 2017). Its distance is $306.0 \pm 1.8 \mathrm{pc}$ (Gaia Collaboration, 2018). Parameters of the planet are listed in Tab. 1. Southworth et al. (2012) studied HAT-P-5 using ground-based follow-up observations. They obtained similar results to Bakos et al. (2007). 

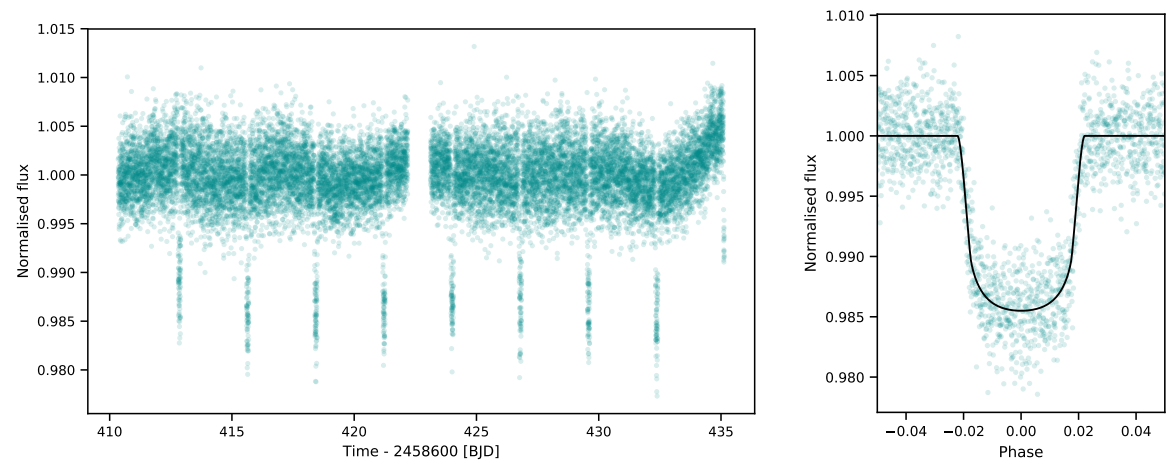

Figure 1. TESS light-curve (left) and phase curve (right) of HAT-P-5 b. Black curve corresponds to model created with PyTransit.
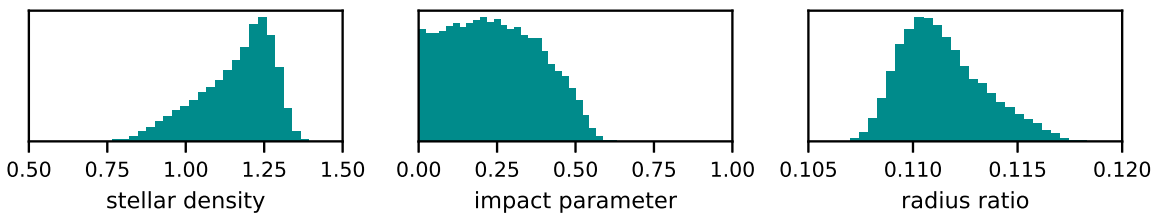

Figure 2. Posterior distributions of fitted parameters of HAT-P-5 b transit.

TESS mission observed HAT-P-5 in Sector 26 (from 8 June to 4 July 2020). The nine transit events were observed (see Fig. 1). However, the last transit was observed only partially - only ingress part.

We obtained asymmetric posterior distributions of more fitted parameters from MCMC sampling (Fig. 2). These distributions are not normal (Gaussian). Therefore, we had to calculate the separate values of the upper and lower uncertainties of these parameters. We could also put only the upper limit on the impact parameter $(<0.485)$ for the same reason. Except for this, the obtained values of parameters (Tab. 2) agree with the values from the literature (Tab. 1).

\subsection{HATS-24 b}

HATS-24 b was discovered by HATSouth project in 2017 (Bento et al., 2017). HATSouth survey is a network of 6 astrograph telescope systems designed to detect transiting exoplanets in orbit around relatively bright stars visible from the Southern hemisphere (Bakos et al., 2013). The exoplanet is orbiting F-dwarf star with mass $1.218 \pm 0.036 \mathrm{M}_{\odot}$, radius $1.194{ }_{-0.041}^{+0.066} \mathrm{R}_{\odot}$ and effective temper- 
ature $6346 \pm 81 \mathrm{~K}$ (Bento et al., 2017). HATS-24b is high-mass hot Jupiter and main parameters are listed in Tab. 1.
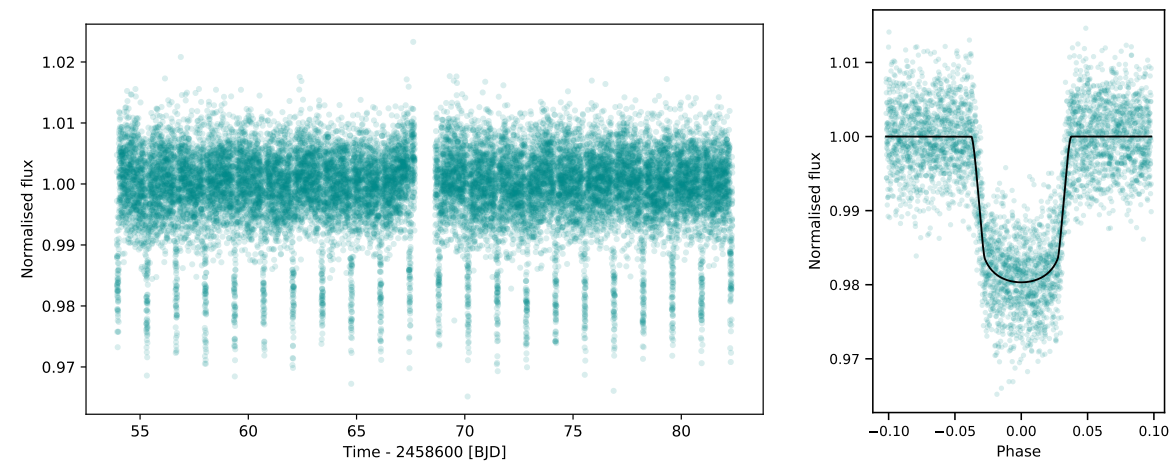

Figure 3. TESS light-curve (left) and phase curve (right) of HATS-24 b. Black curve corresponds to model created with PyTransit.
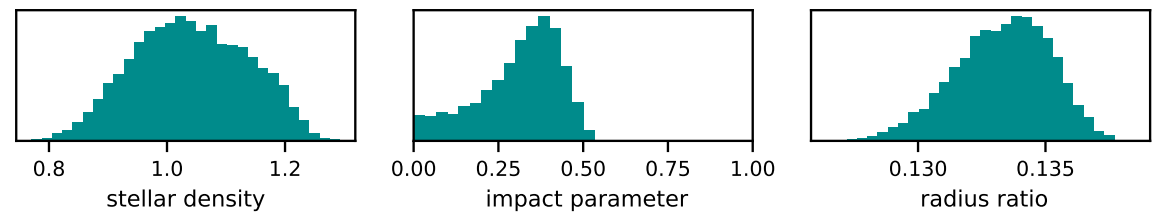

Figure 4. Posterior distributions of fitted parameters of HATS-24 b transit.

TESS observed HATS-24 b in $13^{\text {th }}$ sector and detected 22 transits. Data from TESS are shown in Fig. 3. Using this data we tried to improve parameters. We used PyTransit for fitting and we obtained almost symmetric posterior distributions of fitted parameters (see Fig. 4). In Tab. 2 are listed obtained values from our MCMC fitting. Our results are in good agreement with the values from the literature.

\subsection{HATS-33 b}

HATS-33 b was discovered by de Val-Borro et al. (2016) in the boundaries of the HATSouth survey. HATS-33 b planet was classified as typical hot Jupiter with period $2.549 \mathrm{~d}$ and radius $1.23 \mathrm{R}_{\mathrm{Jup}}$. Main parameters of the planet are listed in Tab. 1. 

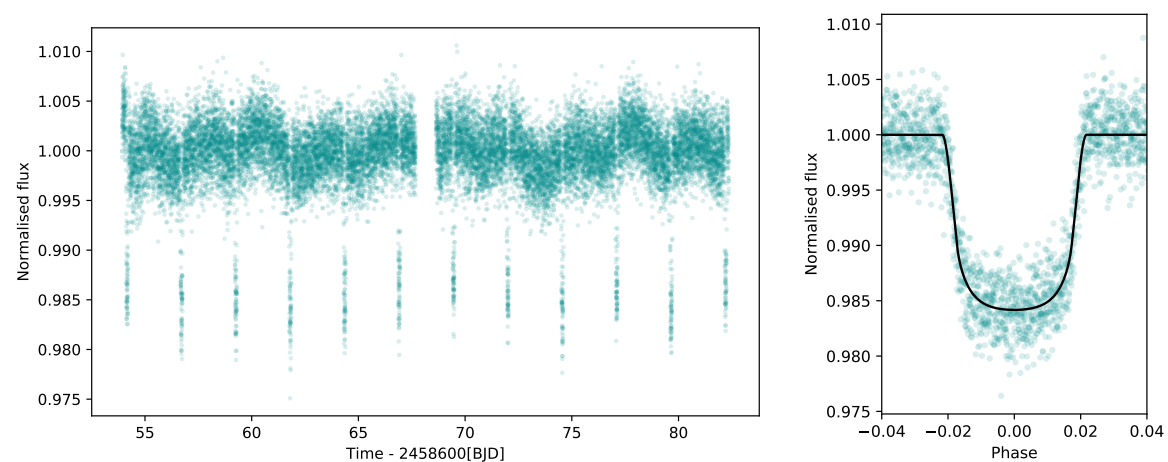

Figure 5. TESS light-curve (left) and phase curve (right) of HATS-33 b. Black curve corresponds to model created with PyTransit.
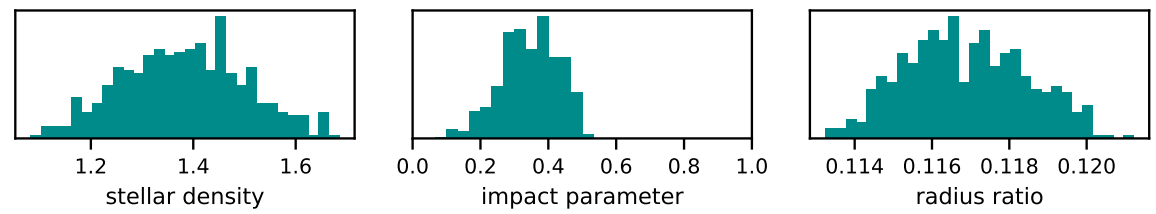

Figure 6. Posterior distributions of fitted parameters of HATS-33 b transit.

The host star is a sun-like star with effective temperature $5659 \pm 85 \mathrm{~K}$, radius $1.022 \pm 0.05 \mathrm{R}_{\odot}$ and mass $1.062 \pm 0.032 \mathrm{M}_{\odot}$ (de Val-Borro et al., 2016). HATS-33 b was observed by TESS in $13^{\text {th }}$ sector. With this new data, we tried to improve exoplanet parameters. The model of transit created with PyTransit is displayed in Fig. 5. New parameters of exoplanet are listed in Tab. 2. We obtained almost symmetric posterior distributions of most of the fitted parameters from MCMC sampling (Fig. 6). These distributions are close to normal (Gaussian). PyTransit allowed us to obtain 10 times better precision for the orbital period and 5 times better precision for radius ratio. In general, obtained values of parameters (Tab. 2) are in good agreement with the values from the literature.

\section{Summary}

We used photometric data collected by space mission TESS to redetermine the basic parameters of three exoplanets HAT-P-5 b, HATS-33 b and HATS$24 \mathrm{~b}$. TESS provides data with high precision and high cadence. It allows to 
Table 1. Parameters of exoplanets and parent stars from literature: $R_{\star}$ - radius of the star, $M_{\star}$ - mass of the star, $T_{\text {eff }}$ - effective temperature of the star, $t_{\mathrm{c}}$ - transit epoch (BJD - 2450000), $p$ - orbital period, $\Delta$ - transit depth, $b$ - impact parameter, $\rho_{\star}$ - stellar density, $q_{1}, q_{2}$ - coefficients of quadratic limb-darkening (given by Claret 2017), $a$ - semi-major axis, $i$ - orbital inclination, $k$ - radius ratio, $r_{\mathrm{p}}$ - planet radius.

\begin{tabular}{c|ccc}
\hline \hline & HAT-P-5 b ${ }^{(1,2)}$ & HATS-24 b ${ }^{(3,4)}$ & HATS-33 b ${ }^{(5)}$ \\
\hline$R_{\star}\left[\mathrm{R}_{\odot}\right]$ & $1.12 \pm 0.09$ & $1.1944_{-0.041}^{+0.066}$ & $1.022 \pm 0.05$ \\
$M_{\star}\left[\mathrm{M}_{\odot}\right]$ & $1.04 \pm 0.29$ & $1.218 \pm 0.036$ & $1.062 \pm 0.032$ \\
$T_{\text {eff }}[\mathrm{K}]$ & $5960 \pm 100$ & $6346 \pm 81$ & $5659 \pm 85$ \\
\hline$t_{\mathrm{c}}[\mathrm{BJD}]$ & $4241.7766 \pm 2 \cdot 10^{-4}$ & $7948.709321 \pm 4 \cdot 10^{-5}$ & $6497.23181 \pm 5 \cdot 10^{-4}$ \\
$p[\mathrm{~d}]$ & $2.7884910 \pm 2.5 \cdot 10^{-5}$ & $1.3484954 \pm 1.3 \cdot 10^{-6}$ & $2.5495551 \pm 6.1 \cdot 10^{-6}$ \\
$\Delta[\%]$ & $1.240 \pm 0.013$ & $1.638 \pm 0.004$ & $1.530 \pm 0.198$ \\
$b\left[R_{\star}\right]$ & $0.425 \pm 0.048$ & - & $0.33 \pm 0.12$ \\
$\rho_{\star}\left[\mathrm{g} / \mathrm{cm}^{3}\right]$ & $1.03 \pm 0.18$ & - & $1.42 \pm 0.17$ \\
$q_{1}$ & 0.275 & 0.240 & 0.342 \\
$q_{2}$ & 0.294 & 0.299 & 0.260 \\
\hline$a\left[R_{\star}\right]$ & $7.50 \pm 0.19$ & $4.561 \pm 0.029$ & $7.843 \pm 0.078$ \\
$a[\mathrm{au}]$ & $0.04075 \pm 0.00076$ & $0.02380 \pm 0.00100$ & $0.03727 \pm 0.00037$ \\
$i\left[{ }^{\circ}\right]$ & $86.75 \pm 0.44$ & $85.97 \pm 0.26$ & $87.62 \pm 0.92$ \\
$k[-]$ & $0.1106 \pm 0.0006$ & $0.12801 \pm 0.00017$ & $0.1237 \pm 0.0080$ \\
$r_{\mathrm{p}}\left[\mathrm{R}_{\mathrm{jup}}\right]$ & $1.260 \pm 0.050$ & $1.395 \pm 0.057$ & $1.230 \pm 0.112$ \\
\hline \hline
\end{tabular}

Source: (1) Bakos et al. (2007), (2) Stassun et al. (2017), (3) Bento et al. (2017), (4) Oliveira et al. (2019), (5) de Val-Borro et al. (2016).

Table 2. New parameters of exoplanets. For detail description see Table 1.

\begin{tabular}{c|ccc}
\hline \hline & HAT-P-5 b & HATS-24 b & HATS-33 b \\
\hline$t_{\mathrm{c}}[\mathrm{BJD}]$ & $5432.4551 \pm 5 \cdot 10^{-4}$ & $7038.47339 \pm 3.7 \cdot 10^{-4}$ & $6497.2318 \pm 5 \cdot 10^{-4}$ \\
$p[\mathrm{~d}]$ & $2.7884732 \pm 4 \cdot 10^{-7}$ & $1.34849750 \pm 3.3 \cdot 10^{-7}$ & $2.549564 \pm 6.2 \cdot 10^{-7}$ \\
$\Delta[\%]$ & $1.235_{-0.036}^{+0.054}$ & $1.779 \pm 0.051$ & $1.346 \pm 0.035$ \\
$b\left[R_{\star}\right]$ & $<0.485$ & $0.34 \pm 0.12$ & $0.35 \pm 0.08$ \\
$\rho_{\star}\left[\mathrm{g} / \mathrm{cm}^{3}\right]$ & $1.18_{-0.16}^{+0.09}$ & $1.039 \pm 0.096$ & $1.38 \pm 0.12$ \\
$q_{1}$ & $0.482_{0.235}^{0.214}$ & $0.170 \pm 0.152$ & $0.565 \pm 0.210$ \\
$q_{2}$ & $0.170_{-0.104}^{+0.214}$ & $0.299 \pm 0.264$ & $0.144 \pm 0.130$ \\
\hline$a\left[R_{\star}\right]$ & $7.86_{-0.36}^{+0.20}$ & $4.64 \pm 0.14$ & $7.85 \pm 0.28$ \\
$a[\mathrm{au}]$ & $0.04097_{-0.00097}^{+0.00189}$ & $0.02576 \pm 0.00078$ & $0.03731 \pm 0.00133$ \\
$i\left[{ }^{\circ}\right]$ & $>86.16$ & $85.82 \pm 1.58$ & $87.55 \pm 1.03$ \\
$k[-]$ & $0.1111_{-0.0017}^{+0.0028}$ & $0.1334 \pm 0.0019$ & $0.1160 \pm 0.0015$ \\
$r_{\mathrm{p}}\left[\mathrm{R}_{\mathrm{jup}}\right]$ & $1.211_{-0.026}^{+0.026}$ & $1.550 \pm 0.022$ & $1.154 \pm 0.015$ \\
\hline \hline
\end{tabular}


characterise the transiting planets in more details than from ground-based observations. Time span of one TESS sector is only 27.4 days but it includes several transits of short-period planet such as hot Jupiters. The exposure time of TESS data (2 $\mathrm{min})$ is short enough, therefore the brightness of the occulted star does not change significantly during this time and the shape of transit is not deformed.

We confirmed the basic parameters of studied planets given in the literature. We improved the values of many of them. We used MCMC method to estimate their uncertainties which are very reliable. In the case of HAT-P-5 b, we obtained very asymmetric posterior distributions of a few parameters. And for the impact parameter, we could only put an upper limit of it. In other studied planets, the distributions were close to normal (Gaussian) distribution. Obtained values of limb-darkening coefficients do not fully agree with the values calculated by Claret (2017). One reason for these differences could be the large step of stellar parameters in the calculated values. Our coefficients have also large uncertainties. We observed the strong correlations between coefficients $q_{1}$ and $q_{2}$ which could affect our results. However, another explanation of our large uncertainties may be the fact that even a large change of limb-darkening coefficients has minimal effect on the shape of transit.

Our results show that TESS data should be used to study already discovered transiting exoplanets to improve their parameters.

Acknowledgements. The authors acknowledge the support from ERASMUS+ grant number 2017-1-CZ01-KA203-035562. The research of PG was supported by internal grant VVGS-PF-2019-1386 of the Faculty of Science, P. J. Šafárik University in Košice. The work of AM was supported by the Slovak Scientific Grant Agency VEGA $2 / 0031 / 18$.

\section{References}

Bakos, G., Noyes, R. W., Kovács, G., et al., Wide-Field Millimagnitude Photometry with the HAT: A Tool for Extrasolar Planet Detection. 2004, Publications of the $A S P$, 116, 266, DOI: $10.1086 / 382735$

Bakos, G. Á., Csubry, Z., Penev, K., et al., HATSouth: A Global Network of Fully Automated Identical Wide-Field Telescopes. 2013, Publications of the ASP, 125, 154, DOI: $10.1086 / 669529$

Bakos, G. Á., Shporer, A., Pál, A., et al., HAT-P-5b: A Jupiter-like Hot Jupiter Transiting a Bright Star. 2007, Astrophysical Journal, Letters, 671, L173, DOI: $10.1086 / 525022$

Bento, J., Schmidt, B., Hartman, J. D., et al., HATS-22b, HATS-23b and HATS-24b: three new transiting super-Jupiters from the HATSouth project. 2017, Monthly Notices of the $R A S, \mathbf{4 6 8}, 835$, DOI: $10.1093 / \mathrm{mnras} / \mathrm{stx} 500$ 
Claret, A., Limb and gravity-darkening coefficients for the TESS satellite at several metallicities, surface gravities, and microturbulent velocities. 2017, Astronomy and Astrophysics, 600, A30, DOI: 10.1051/0004-6361/201629705

de Val-Borro, M., Bakos, G. Á., Brahm, R., et al., HATS-31b through HATS-35b: Five Transiting Hot Jupiters Discovered By the HATSouth Survey. 2016, Astronomical Journal, 152, 161, DOI: 10.3847/0004-6256/152/6/161

Foreman-Mackey, D., Agol, E., Ambikasaran, S., \& Angus, R., Fast and Scalable Gaussian Process Modeling with Applications to Astronomical Time Series. 2017, Astronomical Journal, 154, 220, DOI: 10.3847/1538-3881/aa9332

Gaia Collaboration, Gaia Data Release 2. Summary of the contents and survey properties. 2018, Astronomy and Astrophysics, 616, A1, DOI: 10.1051/0004$6361 / 201833051$

Gilbert, E. A., Barclay, T., Schlieder, J. E., et al., The First Habitable-zone Earthsized Planet from TESS. I. Validation of the TOI-700 System. 2020, Astronomical Journal, 160, 116, DOI: 10.3847/1538-3881/aba4b2

Giménez, A., Equations for the analysis of the light curves of extra-solar planetary transits. 2006, Astronomy and Astrophysics, 450, 1231, DOI: 10.1051/00046361:20054445

Huang, C. X., Burt, J., Vanderburg, A., et al., TESS Discovery of a Transiting SuperEarth in the pi Mensae System. 2018, Astrophysical Journal, Letters, 868, L39, DOI: $10.3847 / 2041-8213 /$ aaef91

Mandel, K. \& Agol, E., Analytic Light Curves for Planetary Transit Searches. 2002, Astrophysical Journal, Letters, 580, L171, DOI: 10.1086/345520

Maxted, P. F. L. \& Gill, S., qpower2: A fast and accurate algorithm for the computation of exoplanet transit light curves with the power-2 limb-darkening law. 2019, Astronomy and Astrophysics, 622, A33, DOI: 10.1051/0004-6361/201834563

Oliveira, J. M., Martioli, E., \& Tucci-Maia, M., First Observation of a Planetary Transit with the SPARC4 CCD: Improved Parameters for HATS-24b. 2019, Research Notes of the American Astronomical Society, 3, 35, DOI: 10.3847/2515-5172/ab06c7

Parviainen, H., PYTRANSIT: fast and easy exoplanet transit modelling in PYTHON. 2015, Monthly Notices of the RAS, 450,3233, DOI: 10.1093/mnras/stv894

Parviainen, H., RoadRunner: a fast and flexible exoplanet transit model. 2020, Monthly Notices of the RAS, 499, 1633, DOI: 10.1093/mnras/staa2901

Penteado, E. M., Rivero-Gonzalez, J., \& Downer, B., IAU100 NameExoWorlds: A Call to Promote Global Citizenship. 2019, Communicating Astronomy with the Public Journal, 26, 5

Ricker, G. R., Winn, J. N., Vanderspek, R., et al., Transiting Exoplanet Survey Satellite (TESS). 2014, in Society of Photo-Optical Instrumentation Engineers (SPIE) Conference Series, Vol. 9143, Space Telescopes and Instrumentation 2014: Optical, Infrared, and Millimeter Wave, 914320

Southworth, J., Mancini, L., Maxted, P. F. L., et al., Physical properties and radius variations in the HAT-P-5 planetary system from simultaneous four-colour 
photometry. 2012, Monthly Notices of the RAS, 422, 3099, DOI: 10.1111/j.13652966.2012.20828.x

Stassun, K. G., Collins, K. A., \& Gaudi, B. S., Accurate Empirical Radii and Masses of Planets and Their Host Stars with Gaia Parallaxes. 2017, Astronomical Journal, 153, 136, DOI: $10.3847 / 1538-3881 /$ aa5df3

Vanderburg, A., Rappaport, S. A., Xu, S., et al., A Giant Planet Candidate Transiting a White Dwarf. 2020, Nature, 585, 363, DOI: 10.1038/s41586-020-2713-y 